\title{
Atmospheric channel characteristics for quantum communication with continuous polarization variables
}

\author{
B. Heim • D. Elser · T. Bartley • M. Sabuncu • \\ C. Wittmann · D. Sych • C. Marquardt • G. Leuchs
}

Received: 24 August 2009 / Revised version: 7 November 2009 / Published online: 11 December 2009

(C) The Author(s) 2009. This article is published with open access at Springerlink.com

\begin{abstract}
We investigate the properties of an atmospheric channel for free space quantum communication with continuous polarization variables. In our prepare-and-measure setup, coherent polarization states are transmitted through an atmospheric quantum channel of $100 \mathrm{~m}$ length on the flat roof of our institute's building. The signal states are measured by homodyne detection with the help of a local oscillator (LO) which propagates in the same spatial mode as the signal, orthogonally polarized to it. Thus the interference of signal and LO is excellent and atmospheric fluctuations are auto-compensated. The $\mathrm{LO}$ also acts as a spatial and spectral filter, which allows for unrestrained daylight operation. Important characteristics for our system are atmospheric channel influences that could cause polarization, intensity and position excess noise. Therefore we study these influences in detail. Our results indicate that the channel is suitable for our quantum communication system in most weather conditions.
\end{abstract}

B. Heim $(\bowtie) \cdot$ D. Elser · T. Bartley · M. Sabuncu · C. Wittmann ·

D. Sych · C. Marquardt · G. Leuchs

Max Planck Institute for the Science of Light,

Günther-Scharowsky-Str. 1, Bau 24, 91058 Erlangen, Germany

e-mail: bettina.heim@mpl.mpg.de

Fax: +49-9131-6877199

B. Heim · D. Elser · T. Bartley · M. Sabuncu · C. Wittmann

D. Sych $\cdot$ C. Marquardt $\cdot$ G. Leuchs

Institute of Optics, Information and Photonics, University

of Erlangen-Nuremberg, Staudtstr. 7/B2, 91058 Erlangen,

Germany

T. Bartley

Clarendon Laboratory, University of Oxford, Parks Road, Oxford

OX1 3PU, England, UK

\section{Introduction}

Quantum communication describes the distribution of quantum states between two parties, traditionally named Alice and Bob. These states can for example be entangled [1] states, providing the basis for various protocols such as quantum teleportation [2] or quantum dense coding [3]. Many of the initial research projects used discrete quantum variables. Later also continuous variables have proven suitable for quantum communication (for a review see [4]).

Quantum key distribution (QKD) $[5,6]$ is a further important branch of quantum communication and concerns the establishment of a secret key jointly between Alice and Bob with the help of a quantum channel. The security is based on the laws of quantum mechanics. In principle unconditional security can be achieved. Any two non-orthogonal quantum states suffice to ensure secure key distribution [7]. This holds as long as the detection matches the quantum state emitted by the source. A single-photon detector e.g. matches weak coherent states as long as the probability for multiphoton events is low enough. For higher multiphoton probabilities [8] or even bright polarization states $[9,10]$ a singlephoton detector can not be used. In such scenarios, however, photon number resolving detectors, homodyne or heterodyne detectors are a better match, promising unconditionally secure key distribution.

Free space QKD over an atmospheric channel was first demonstrated in 1996 [11]. Since then, a number of prepareand-measure as well as entanglement based schemes have been implemented in free space (for a review see [6]). The current world record in distance is $144 \mathrm{~km}[12,13]$ and satellite quantum communication is already in preparation $[14,15]$. All of these systems use single-photon detectors and therefore have to employ spatial, spectral and/or temporal filtering in order to reduce background light. In our system, we use an alternative approach: with the help of a bright 
local oscillator (LO), we perform homodyne measurements on weak coherent polarization states [10]. We focus on the characterization of the quantum channel, which is a $100 \mathrm{~m}$ free space link on the roof of our institute's building.

In classical free space communication systems using homodyne detection (e.g. [16]), producing the LO locally at the receiver is appropriate. In QKD, on the other hand, the requirements for detection efficiency are more stringent, as fragile quantum states are transmitted. Thus we developed a protocol using the polarization degree of freedom to multiplex signal and LO [9]. The LO is produced by Alice and propagates in the same spatial channel mode as the signal.

In quantum mechanics, polarization is conveniently described by the quantum Stokes operators, that are the quantum counterpart of the classical Stokes parameters [17]. The Stokes operators are introduced and defined for example in [18]. In a homodyne detection of the Stokes operators, the co-propagation of signal and LO leads to an intrinsically excellent spatial interference between the two. This translates to a high detection efficiency without any additional interference stabilization. For our free space system, there are also advantageous side effects of this co-propagation: firstly, the LO acts as a spatial filter, such that only those photons, that are spatially mode-matched to it will result in a significant detector signal. Unlike in single-photon experiments there is no need for spatial filtering by pinholes or fibers. Secondly, the LO facilitates spectral filtering, as the beat-note of signal and LO, interfering at a polarizing beam splitter (PBS), can be electronically filtered at the detector. The detection bandwidth can thus be adjusted precisely and background light outside this range does not disturb the measurement. Finally, absolute phase fluctuations in the channel are autocompensated, as they are identical for signal and LO.

The theory for the propagation of classical light through turbulent atmosphere including diverse phenomena such as beam wander or beam spreading has been investigated in e.g. [19-21]. These investigations are based on work on the description of turbulence by Kolmogorov. The refractive index structure parameter $C_{n}^{2}(h)$ is a measure for the "strength" of optical turbulence due to random variations of the refractive index in the atmosphere (see e.g. [22]). $C_{n}^{2}(h)$ is a function of the wavelength, atmospheric pressure and atmospheric temperature and varies strongly with the height $h$ above ground. Its value can vary from $10^{-17} \mathrm{~m}^{-\frac{2}{3}}$ or less for weak turbulence up to $10^{-12} \mathrm{~m}^{-\frac{2}{3}}$ for strong optical turbulence [22].

The beam radius $w$ of a Gaussian beam after propagating a distance $z$ through turbulent atmosphere is given by [23]:

$w(z)=w_{0} \sqrt{1+\xi \frac{z^{2}}{z_{\mathrm{R}}^{2}}}$, with the Rayleigh length $z_{\mathrm{R}}$, the beam waist $w_{0}$ and the global coherence parameter $\xi=\xi_{\mathrm{S}}+\frac{2 w_{0}^{2}}{\rho_{0}^{2}}$. In this notation, $\rho_{0}=\left(0.55 C_{n}^{2} k^{2} z\right)^{-\frac{3}{5}}$ is the coherence length of a spherical wave propagating in optical turbulence and $\xi_{\mathrm{S}}$ describes the spatial coherence properties of the laser beam $\left(\xi_{\mathrm{S}}=1\right.$ for a coherent beam). In absence of atmospheric turbulence, $\rho_{0} \rightarrow \infty$, the global coherence parameter $\xi$ reduces to unity and thus the evolution of the beam waist $w(z)$ corresponds to a Gaussian beam.

Effects on quantum continuous variable states have only recently been studied in the context of propagation through turbulent atmosphere [10, 24-28]. In security analysis of QKD systems, all excess noise is considered to originate from Eve's interactions. In a worst case scenario, even small noise effects could prevent a key to be established. Intensity noise, for example, can easily stem from the practical issue of finite aperture sizes. Such noise is typically of nonGaussian character (e.g. on-off noise [27]). Squeezed and entangled states that were degraded by non-Gaussian noise can be distilled with Gaussian operations [24, 25].

\section{Experimental setup}

\subsection{Quantum state measurements}

With the setup in Fig. 1 we demonstrated the feasibility of continuous variable atmospheric QKD at daylight [10]. The scheme follows the principles of our earlier laboratory work [9, 29]. We use a grating-stabilized $\mathrm{CW}$ diode laser, whose wavelength of $809 \mathrm{~nm}$ lies within an atmospheric transmission window. A linearly polarized laser beam $\left(\hat{S}_{1}\right.$ in terms of Stokes operators) is emitted by Alice and later serves as a LO in Bob's measurement. A modulator is used to generate the coherent signal states. (A magneto-optical modulator (MOM) for example employs the Faraday effect to tilt the linear polarization by small amounts.) The weak

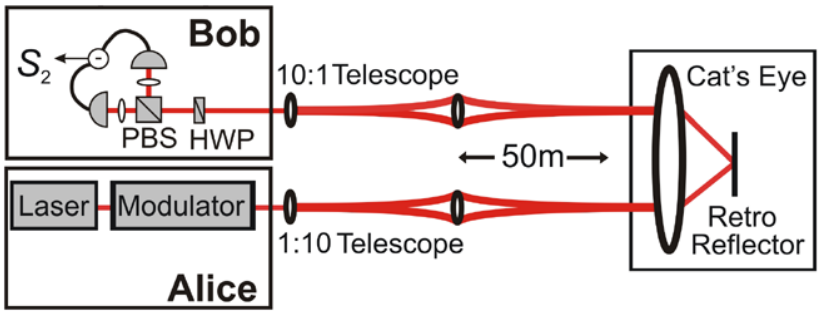

Fig. 1 Experimental setup for our QKD feasibility studies [10]: Alice's laser emits a linearly polarized $\mathrm{CW}$ beam which later serves as a local oscillator (LO) for Bob's measurements. In terms of Stokes operators, the local oscillator is $\hat{S}_{1}$-polarized. Alice's modulator generates a weak signal that Bob then measures by an $\hat{S}_{2}$ Stokes detection. In-between, the beam is expanded and sent to a retro-reflector at a distance of $50 \mathrm{~m}$. After reflection, Bob's telescope again reduces the beam diameter. PBS: polarizing beam splitter, HWP: half wave plate 
signal component of a mean photon number of typically less than one photon per pulse is located in the same spatial mode as the LO, but is polarized orthogonally to it. After expanding the beam by a telescope, the signal/LO beam is sent over the roof of our institute's building and retro-reflected after $50 \mathrm{~m}$. The beam is travelling at a height of less than $1 \mathrm{~m}$ above the roof. Bob reduces the beam diameter with a telescope and then performs a Stokes measurement of the $\hat{S}_{2}$-operator to detect the signal states.

\subsection{Setups for different noise measurements}

There are different methods to estimate and measure the evolution of the $C_{n}^{2}(h)$-parameter, for an overview see e.g. [22]. In our setup, however, we focus on the direct consequences of the atmospheric channel on our QKDexperiment. Thus we did not investigate $C_{n}^{2}(h)$ but instead measured the polarization, position and intensity excess noise in the atmospheric channel.

\subsubsection{Atmospheric polarization noise}

In previous work $[10,30,31]$ we investigated the polarization excess noise introduced by the channel. For an alphabet using two coherent polarization states we compared the distributions of $\hat{S}_{2}$-Stokes measurements of the signal states before and after transmission through the channel. Additional polarization noise introduced by the channel would broaden the measurement distribution. The work in $[30,31]$ showed, that this is not the case. Measurements of the RF frequency spectrum of unmodulated beams that were sent through the atmosphere also allow us to identify the frequency range above $10 \mathrm{kHz}$ to be essentially noiseless [10].

\subsubsection{Atmospheric intensity noise}

Atmospheric intensity noise can be measured by direct detection of the beam. For calibration, we compare the noise of a beam sent through the atmosphere with a beam sent over the optical table. The intensity noise is recorded by a spectrum analyzer. These measurements are sensitive to fluctuations of the laser's intrinsic excess noise which we controlled to be close to zero when recording the spectra. We use low noise detectors whose electronic noise is significantly smaller than the shot noise, thus allowing us to measure at the quantum-noise limit.

\subsubsection{Atmospheric beam jitter}

We used a beam profiling system to measure the changes of the spatial beam profile caused by the atmospheric channel. We compared the profiles of the outgoing and the returning beam with the help of a CCD camera (Metrolux

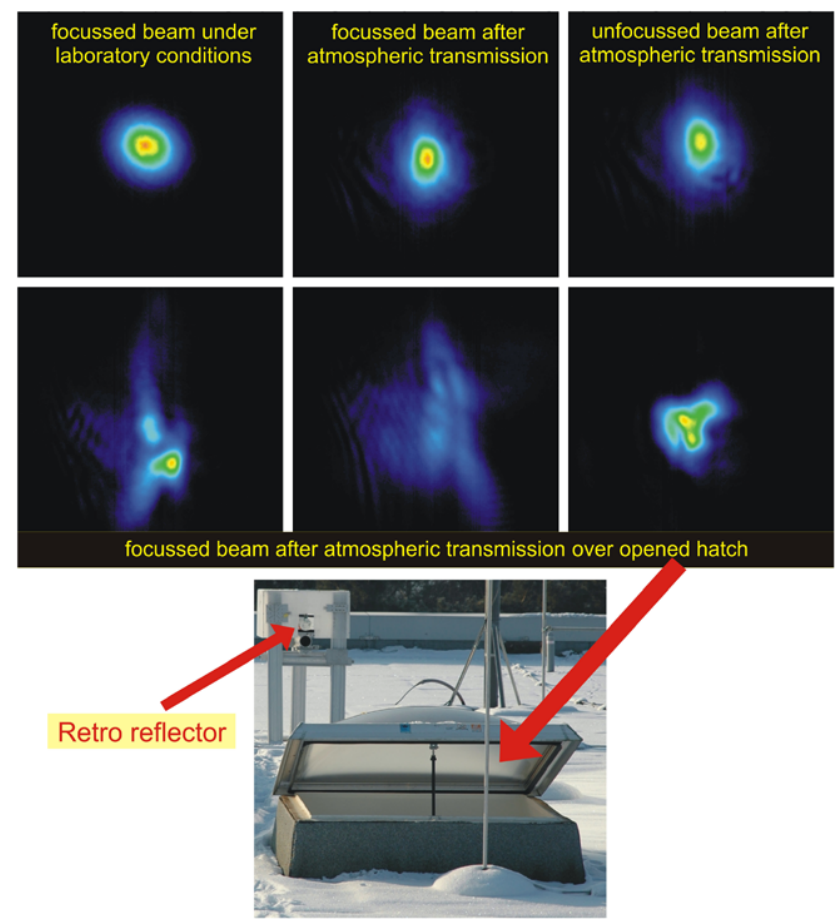

Fig. 2 2D-beam profiles under several conditions. Before being sent over the roof, the beam is in a near TEM $_{00}$ mode (upper left picture). After transmission through the channel the beam profiles are slightly distorted, but the intensity distributions along the two main beam axis are still approximately Gaussian. Strong beam distortions are caused by opening a hatch over which the beam passes on its way to and back from the retro-reflector. Plots thereof, recorded at different instances of time, are shown in the second row. All beam profiles were recorded at an exposure time of $20 \mu \mathrm{s}$

ML3743). The pictures then were analyzed by the Metrolux BeamLux II software package. Figure 2 shows some typical spatial beam profiles under different conditions. Sequences of pictures were taken at an exposure time of $20 \mu \mathrm{s}$.

\section{Results and discussion}

In the following we will concentrate on atmospheric intensity noise and beam jitter (atmospheric polarization noise has been investigated in $[10,30,31])$.

\subsection{Atmospheric intensity noise}

Measurements of the intensity noise were performed by detecting the amplified photocurrents of one photodiode (Hamamatsu S3399, active area $7 \mathrm{~mm}^{2}$, diameter $3 \mathrm{~mm}$ ), and comparing the spectrum of an unmodulated beam transmitted through the atmosphere with that of a reference beam over the table. Constant attenuation was compensated for by setting the optical power in front of the photodiode to the same value of $650 \mu \mathrm{W}$ in both cases. In Fig. 3, it can be seen that there is no atmospheric excess noise measured for 


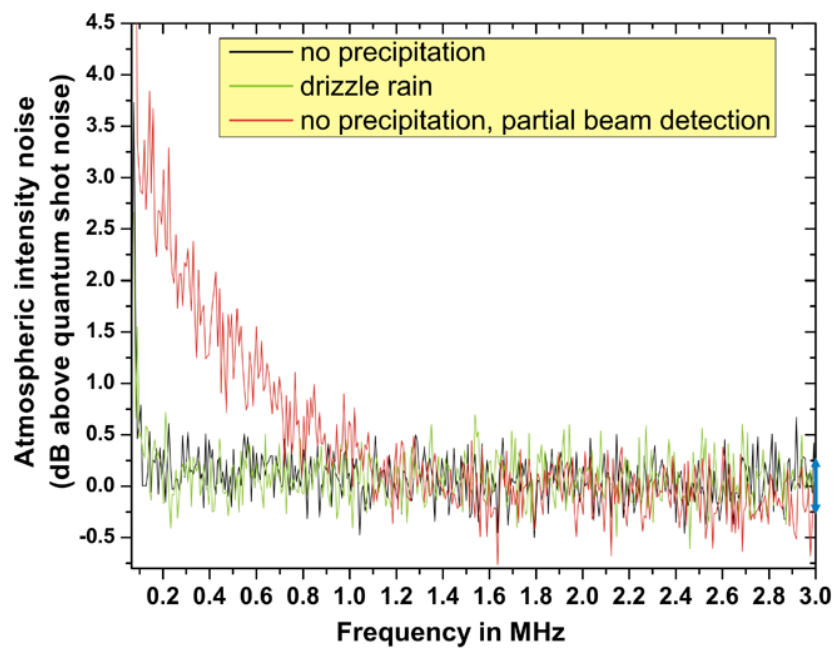

Fig. 3 Intensity noise measured by a direct detection. All curves arise from an averaging over several measurements and are normalized to a quantum-noise limited reference beam. The resolution bandwidth for the measurements consisting of 401 points was $10 \mathrm{kHz}$, the video bandwidth $100 \mathrm{~Hz}$. On the right hand side of the plot, the measurement accuracy of $5 \%$ is shown in blue

beams that are sent through the atmospheric channel in good weather conditions (dry and sunny) as well as in light rain. This is valid for frequencies above the current QKD modulation frequency $(1 \mathrm{MHz})$. The measurement accuracy of $5 \%$ accommodates the fact that the beam moves on regions of the photodiode with slightly different sensitivities. Additionally, small intensity fluctuations of the laser are included. We can infer that the scattering effects of the atmosphere do not cause a measurable beam broadening or spatial beam jitter. Thus, the beam always hits the photodiodes completely as in the case of laboratory conditions.

Intensity excess noise can occur if the collimated beam after Bob's telescope is detected without focussing it onto the photodiodes. Then the spatial beam jitter caused by the atmosphere exceeds the active area of the photodiodes and thus leads to partial detection noise (red curve in Fig. 3). In our setup, the beam travels less than $1 \mathrm{~m}$ above a flat roof which results in rather big refractive index variations. An estimation of the intensity excess noise based on fluctuations of the beam centers will be given in the next section.

\subsection{Atmospheric beam jitter}

Figure 4 shows the beam centers and standard deviations of sequences of beam profiles (examples of spatial intensity distributions are shown in Fig. 2). Part (a) in Fig. 4 shows the comparison of a beam under laboratory conditions with one after atmospheric transmission. Both beams were focussed on the camera when recorded. As expected, the fluctuations of the beam centers are much higher for atmospheric transmission, but still small enough that the beam always com- pletely impinges on the photodiodes. In this case no intensity excess noise is shown in Fig. 3.

We estimate the relative quantum shot noise of these states by $\frac{\sqrt{\langle n\rangle}}{\langle n\rangle}=2 \times 10^{-7}$. The mean photon number $\langle n\rangle$ of the $650 \mu \mathrm{W}$-beams per measurement period is approximated by dividing the total detected energy per measurement $\left(E_{\text {total }}\right)$ by the energy of one photon of $809 \mathrm{~nm}$ $\left(E_{\text {photon }}\right)$ :

$\langle n\rangle=\frac{E_{\text {total }}}{E_{\text {photon }}}=\frac{P_{\text {opt }} \frac{1}{\text { VBW }}}{2.45 \times 10^{-19} \mathrm{Ws}}=2.65 \times 10^{13}$

where the video bandwidth (VBW) of the RF spectrum analyzer is $100 \mathrm{~Hz}$.

By a numerical evaluation we estimate the atmospherically induced intensity noise for a focussed detection. The calculations are based on the measured beam center fluctuations and on values for beam diameters that we also gained from the spatial beam profiles at a frequency of $50 \mathrm{kHz}$. In our calculation, we integrate over the intensity distributions of beam profiles within a region defined by the size of the photodiodes. The resulting intensity value then is normalized to that of non-cropped beams. We assume a Gaussian intensity distribution, a mean beam diameter of $0.98 \mathrm{~mm}$ and aligning inaccuracies of $0.2 \mathrm{~mm}$ are also included in our calculations.

Using the calculations explained above, we compare a centered beam with one whose center is shifted by $0.0134 \mathrm{~mm}$, the mean standard deviation of the beam center fluctuations (see Fig. 4(a)). After normalization to the intensity within the size of the photodiodes, the result is a relative intensity noise around $7 \times 10^{-8}$. This value lies below the quantum shot noise estimated above, that marks the quantum mechanical limitation of our measurement accuracy. Thus it is too small to be detected, in agreement with Fig. 3.

As quoted in Sect. 3.1 and shown in Fig. 3, intensity noise can occur by a detection of an unfocussed beam directly after Bob's telescope. This beam is broadened compared to a focussed one and its beam center fluctuations are slightly higher as shown in Fig. 4(b). We perform the same evaluation as for the focussed beam above, resulting in an intensity noise of $4.4 \times 10^{-7}$. This noise at $50 \mathrm{kHz}$ is about twice the estimated value for the relative quantum noise $\left(2 \times 10^{-7}\right)$. This is in agreement with Fig. 3, showing the intensity noise for an unfocussed beam to be about $3 \mathrm{~dB}$ higher than shot noise for the lowest measured frequencies at around $80 \mathrm{kHz}$. As the detection bandwidth was limited we could not perform the intensity noise measurements all the way down to $50 \mathrm{kHz}$. We expect a slight further increase of the noise for smaller frequencies. Thus, the estimation is in good over all agreement with the measurements. Even though this effect is small, it is still observable by low-noise 


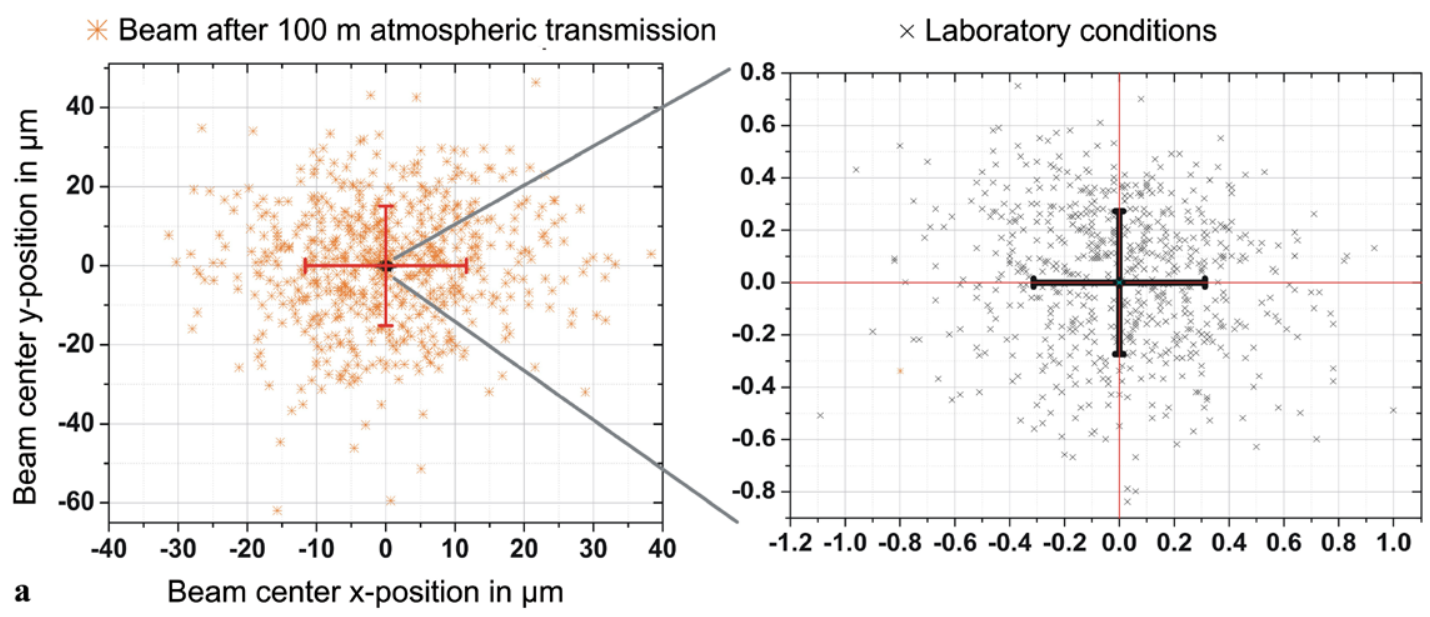

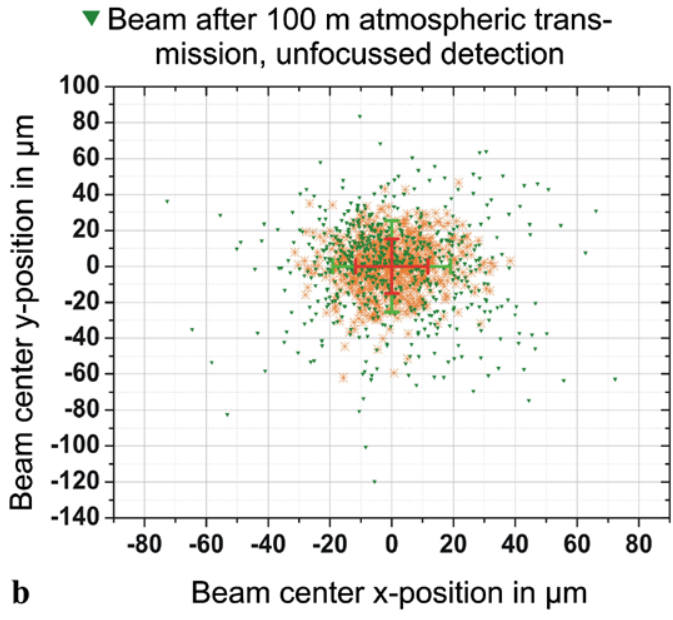

Fig. $4(x, y)$-plots of the beam centers and standard deviations of sequences of 650 beam profiles, recorded with an exposure time of $20 \mu \mathrm{s}$. The mean values of the beam centers are shifted to $(0,0)$ for each plot. The standard deviations are shown in colors corresponding to the particular beam centers. Plot (a) shows the comparison of a beam under laboratory conditions with one that passed through the atmospheric channel, both being focussed on the camera while recorded. As expected, the fluctuations are much larger after atmospheric trans-

detectors exhibiting electronic noise well below the quantum noise.

The beam center fluctuations are even larger when a hatch (see Fig. 2) is open, over which the beam passes on its way to and from the retro-reflector (see Fig. 4(c)). In this particular case, atmospheric fluctuations are dramatically increased. This would cause further intensity noise when the beam is detected at the photodiode. Hence we are now working on an optimised detection system to improve free space beam capture. The use of improved optical tapers can combat strong combined spatial and angular fluctuations of the incident beam, better than a single lens could do [32, 33]. We have experimental evidence for the non-Gaussian character of the noise, which will be reported elsewhere.

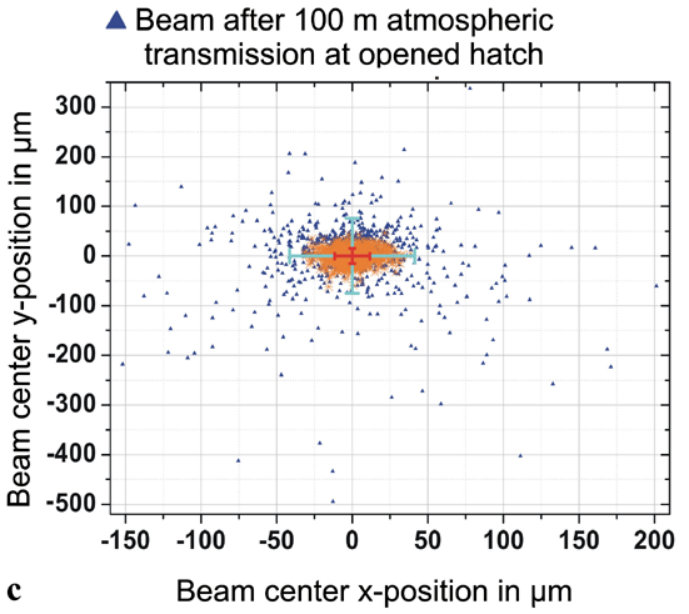

mission. In the lower part, the difference between a focussed and an unfocussed "atmospheric" beam is demonstrated (b), corresponding to the intensity excess noise shown in red in Fig. 3. In (c), we compare this "atmospheric" beam to one having passed directly over a hatch, whereby the temperature gradient between inside the building and outside caused strong atmospheric fluctuations (these measurements were taken during winter time)

\section{Conclusion and outlook}

Within the framework of the first demonstration of continuous variable quantum communication through a real atmospheric channel, we investigated different channel noise properties. We precisely characterized atmospheric intensity fluctuations by quantum-noise limited measurements. Our results indicate that in good weather conditions channel influences like polarization, intensity and position noise are sufficiently low to allow for quantum state transmission and QKD operation at daylight. For our $100 \mathrm{~m}$ link, there was no need for active beam stabilization yet. For the extended link of $1.6 \mathrm{~km}$, on which we are working currently, active stabilization is probably necessary. Monitoring the bright LO can provide us with a control signal for active beam stabi- 
lization. Additionally, to synchronize Alice's and Bob's stations, one can interrupt the $\mathrm{cw}-\mathrm{LO}$ in regular time intervals. Switching on the LO marks the beginning of a new signal frame. This will fulfill the same task as the timing pulses in other free space QKD setups, e.g. [34]. Furthermore, we plan to increase the pulse rate of the quantum states and implement more complex signal alphabets [35] in QKD.

Acknowledgement Denis Sych acknowledges the Alexander von Humboldt Foundation for support through a fellowship.

Open Access This article is distributed under the terms of the Creative Commons Attribution Noncommercial License which permits any noncommercial use, distribution, and reproduction in any medium, provided the original author(s) and source are credited.

\section{References}

1. A. Einstein, B. Podolsky, N. Rosen, Phys. Rev. 47, 777 (1935)

2. C.H. Bennett, G. Brassard, C. Crépeau, R. Jozsa, A. Peres, W.K. Wootters, Phys. Rev. Lett. 70, 1895 (1993)

3. C.H. Bennett, S.J. Wiesner, Phys. Rev. Lett. 69, 2881 (1992)

4. U.L. Andersen, G. Leuchs, C. Silberhorn, Laser Photonics Rev. (2009, in print). doi:10.1002/lpor.200910010

5. N. Gisin, G. Ribordy, W. Tittel, H. Zbinden, Rev. Mod. Phys. 74, 145 (2002)

6. V. Scarani, H. Bechmann-Pasquinucci, N.J. Cerf, M. Dušek, N. Lütkenhaus, M. Peev, Rev. Mod. Phys. 81, 1301 (2009)

7. C.H. Bennett, Phys. Rev. Lett. 68, 3121 (1992)

8. F. Grosshans, G. Van Assche, J. Wenger, R. Brouri, N.J. Cerf, P. Grangier, Nature 421, 6920 (2003)

9. S. Lorenz, N. Korolkova, G. Leuchs, Appl. Phys. B 79, 273 (2004)

10. D. Elser, T. Bartley, B. Heim, C. Wittmann, D. Sych, G. Leuchs, New J. Phys. 11, 045014 (2009)

11. B.C. Jacobs, J.D. Franson, Opt. Lett. 21, 1854 (1996)

12. T. Schmitt-Manderbach, H. Weier, M. Fürst, R. Ursin, F. Tiefenbacher, Th. Scheidl, J. Perdigues, Z. Sodnik, Ch. Kurtsiefer, J.G. Rarity, A. Zeilinger, H. Weinfurter, Phys. Rev. Lett. 98, 010504 (2007)

13. R. Ursin, F. Tiefenbacher, T. Schmitt-Manderbach, H. Weier, T. Scheidl, M. Lindenthal, B. Blauensteiner, T. Jennewein, J. Perdigues, P. Trojek, B. Ömer, M. Fürst, M. Meyenburg, J. Rarity, Z. Sodnik, C. Barbieri, H. Weinfurter, A. Zeilinger, Nat. Phys. 3, 481 (2007)
14. P. Villoresi, T. Jennewein, F. Tamburini, M. Aspelmeyer, C. Bonato, R. Ursin, C. Pernechele, V. Luceri, G. Bianco, A. Zeilinger, C. Barbieri, New J. Phys. 10, 033038 (2008)

15. J.M. Perdigues Armengol, B. Furch, C.J. de Matos, O. Minster, L. Cacciapuoti, M. Pfennigbauer, M. Aspelmeyer, T. Jennewein, R. Ursin, T. Schmitt-Manderbach, G. Baister, J. Rarity, W. Leeb, C. Barbieri, H. Weinfurter, A. Zeilinger, Acta Astronaut. 63, 165 (2008)

16. R. Lange, B. Smutny, B. Wandernoth, R. Czichy, D. Giggenbach, Proc. SPIE 6105, 61050A (2006)

17. G.G. Stokes, Trans. Camb. Philos. Soc. 9, 399 (1852)

18. N. Korolkova, G. Leuchs, R. Loudon, T.C. Ralph, C. Silberhorn, Phys. Rev. A 65, 052306 (2002)

19. V.I. Tatarskii, The effects of the turbulent atmosphere on wave propagation. US Department of Commerce, Springfield, VA, 1971

20. R.L. Fante, Proc. IEEE 63, 1669 (1975)

21. R.L. Fante, Proc. IEEE 68, 1424 (1980)

22. J.C. Ricklin, S.M. Hammel, F.D. Eaton, S.L. Lachinova, J. Opt. Fiber Commun. Rep. 3, 111 (2006)

23. J.C. Ricklin, F.M. Davidson, J. Opt. Soc. Am. A 19, 1794 (2002)

24. J. Heersink, C. Marquardt, R. Dong, R. Filip, S. Lorenz, G. Leuchs, U.L. Andersen, Phys. Rev. Lett. 96, 253601 (2006)

25. R. Dong, M. Lassen, J. Heersink, C. Marquardt, R. Filip, G. Leuchs, U.L. Andersen, Nat. Phys. 4, 919 (2008)

26. B. Hage, A. Samblowski, J. DiGuglielmo, A. Franzen, J. Fiurášek, R. Schnabel, Nat. Phys. 4, 915 (2008)

27. C. Wittmann, D. Elser, U.L. Andersen, R. Filip, P. Marek, G. Leuchs, Phys. Rev. A 78, 032315 (2008)

28. A.A. Semenov, W. Vogel, Phys. Rev. A 80, 021802 (2009)

29. S. Lorenz, J. Rigas, M. Heid, U.L. Andersen, N. Lütkenhaus, G. Leuchs, Phys. Rev. A 74, 042326 (2006)

30. T. Bartley, Free space quantum key distribution with coherent polarisation states. M.Sci. Project, Institute of Optics, Information and Photonics, University of Erlangen-Nuremberg and Blackett Laboratory, Imperial College, London, 2008

31. D. Elser, T. Bartley, B. Heim, C. Wittmann, D. Sych, G. Leuchs, in SECOQC QKD Network Demonstration and International Conference, Vienna, 2008

32. T. Bartley, B. Heim, D. Elser, D. Sych, M. Sabuncu, C. Wittmann, N. Lindlein, C. Marquardt, G. Leuchs, in Proceedings of the QuantumComm 2009, International Conference on Quantum Communication and Quantum Networking, Sorrento Peninsula, Italy. Lecture Notes of ICST (Springer, Berlin, 2009, accepted for publication)

33. R. Winston, J.C. Miñano, P. Benítez, Nonimaging Optics (Elsevier/Academic Press, Amsterdam/San Diego, 2005)

34. R.J. Hughes, J.E. Nordholt, D. Derkacs, C.G. Peterson, New J. Phys. 4, 43 (2002)

35. D. Sych, G. Leuchs, arXiv:0902.1895 [quant-ph] (2009) 